Supporting Information

\title{
Rapid and Sensitive Pathogen Detection by DNA Amplification Using Janus Particle Enabled Rotational Diffusometry
}

\author{
Dhrubajyoti Das ${ }^{\text {a }}$, Wei-Long Chen ${ }^{\text {a }}$, Han-Sheng Chuang *, a, b \\ a Department of Biomedical Engineering, National Cheng Kung University, Tainan 701, Taiwan \\ ${ }^{\mathrm{b}}$ Medical Device Innovation Centre, National Cheng Kung University, Tainan 701, Taiwan \\ * Correspondence: oswaldchuang@ mail.ncku.edu.tw
}

Table of Contents:

1. Supporting data (Figure S1 - Figure S3, Table S1) ........................... S2

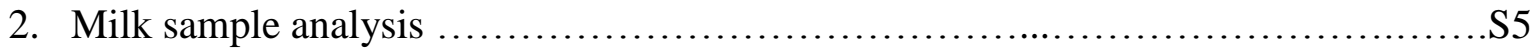

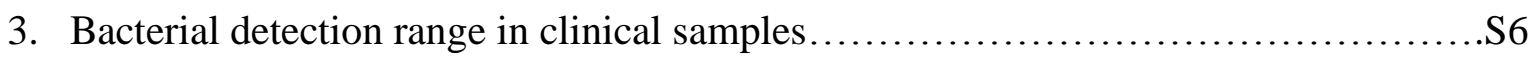

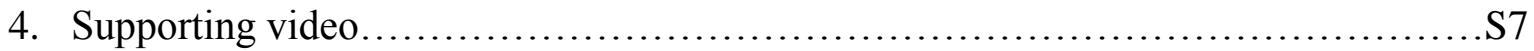

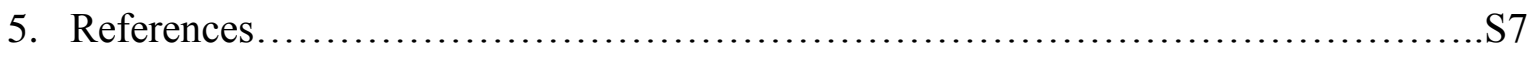




\section{Supporting data:}

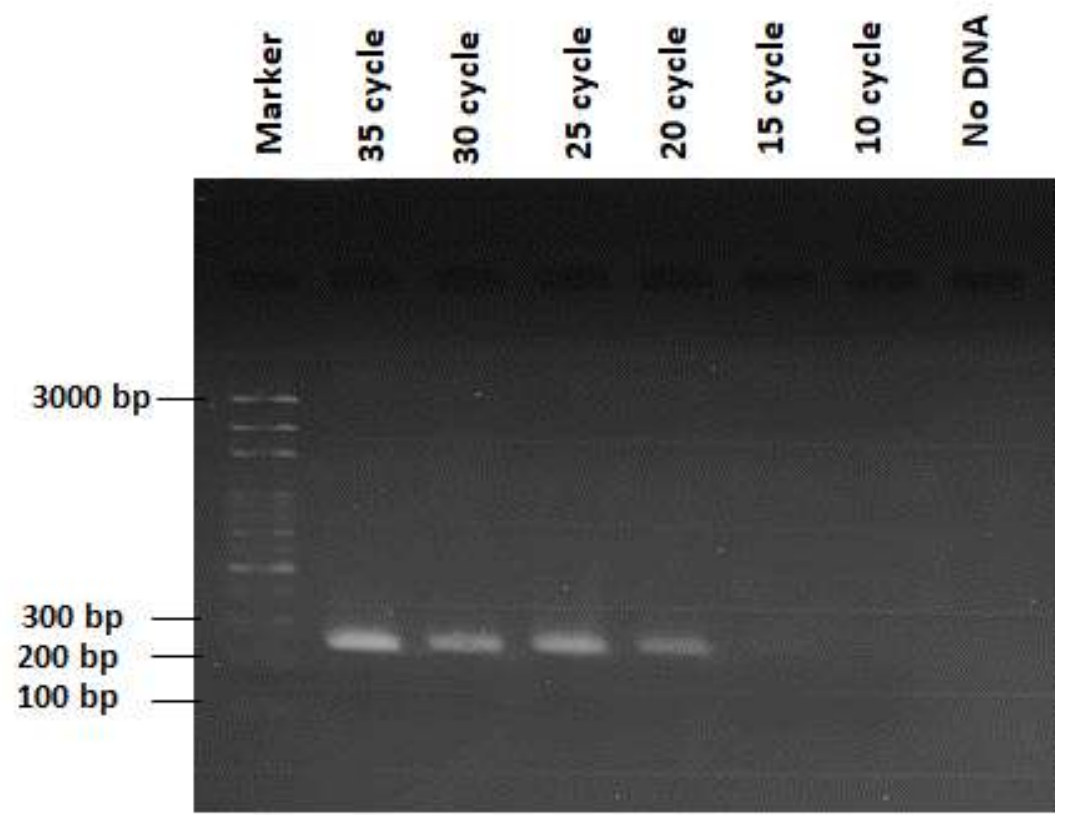

Figure S1. E. coli DNA amplification of uidA gene in different thermal cycles (amplicon size: $246 \mathrm{bp})$ 

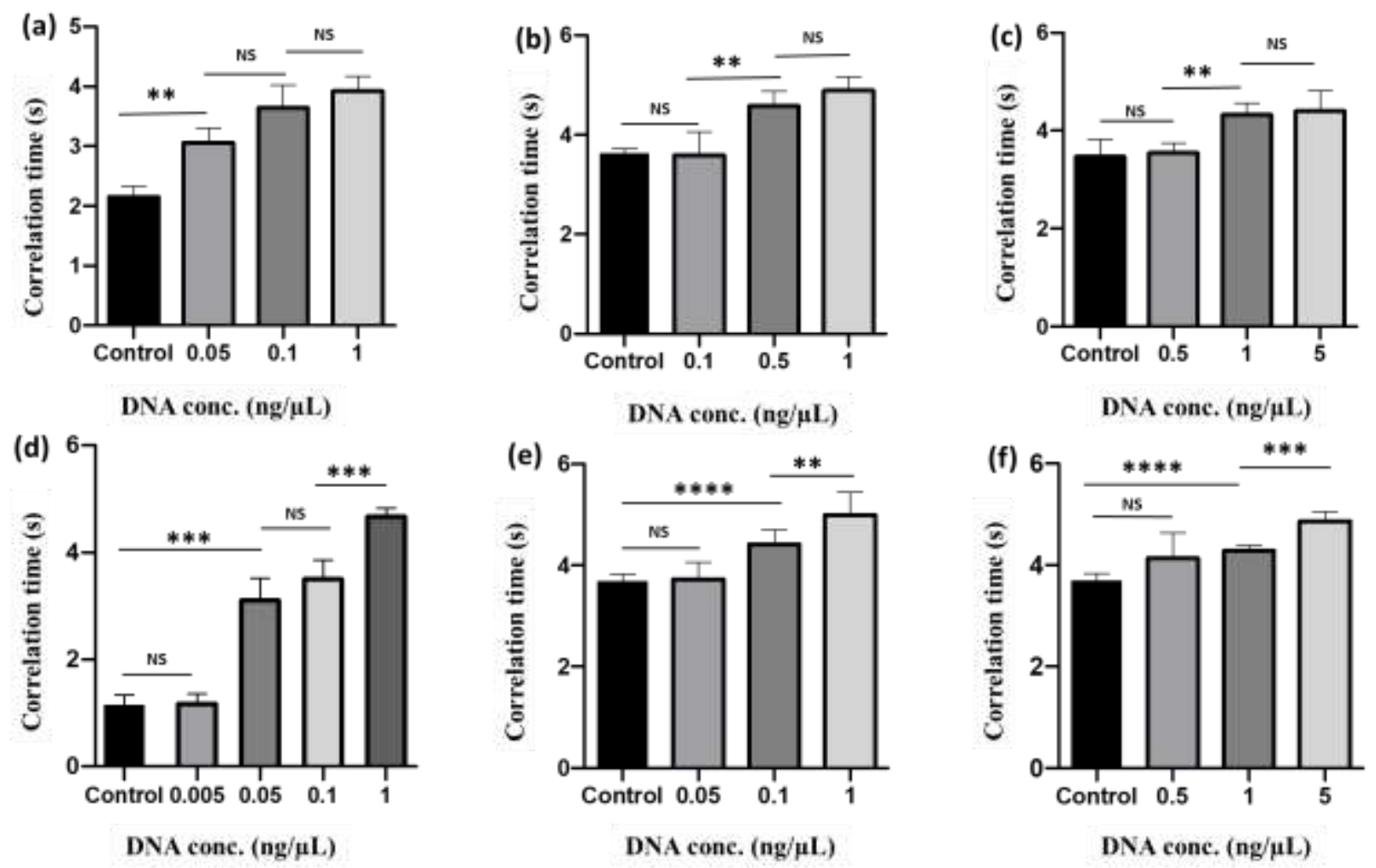

Figure S2. Correlation time plot of PCR amplified DNA of E. coli with respect to template DNA concentration for - 147 bp (a-c) and 246 bp amplicon (d-f) (a) 30 PCR cycles. (b) 20 PCR cycles. (c) 10 PCR cycles. (d) 30 PCR cycles. (e) 20 PCR cycles. (f) 10 PCR cycles. (** p $<0.01$, *** p $<0.001, * * * * \mathrm{p}<0.0001, \mathrm{n}=3)$ 

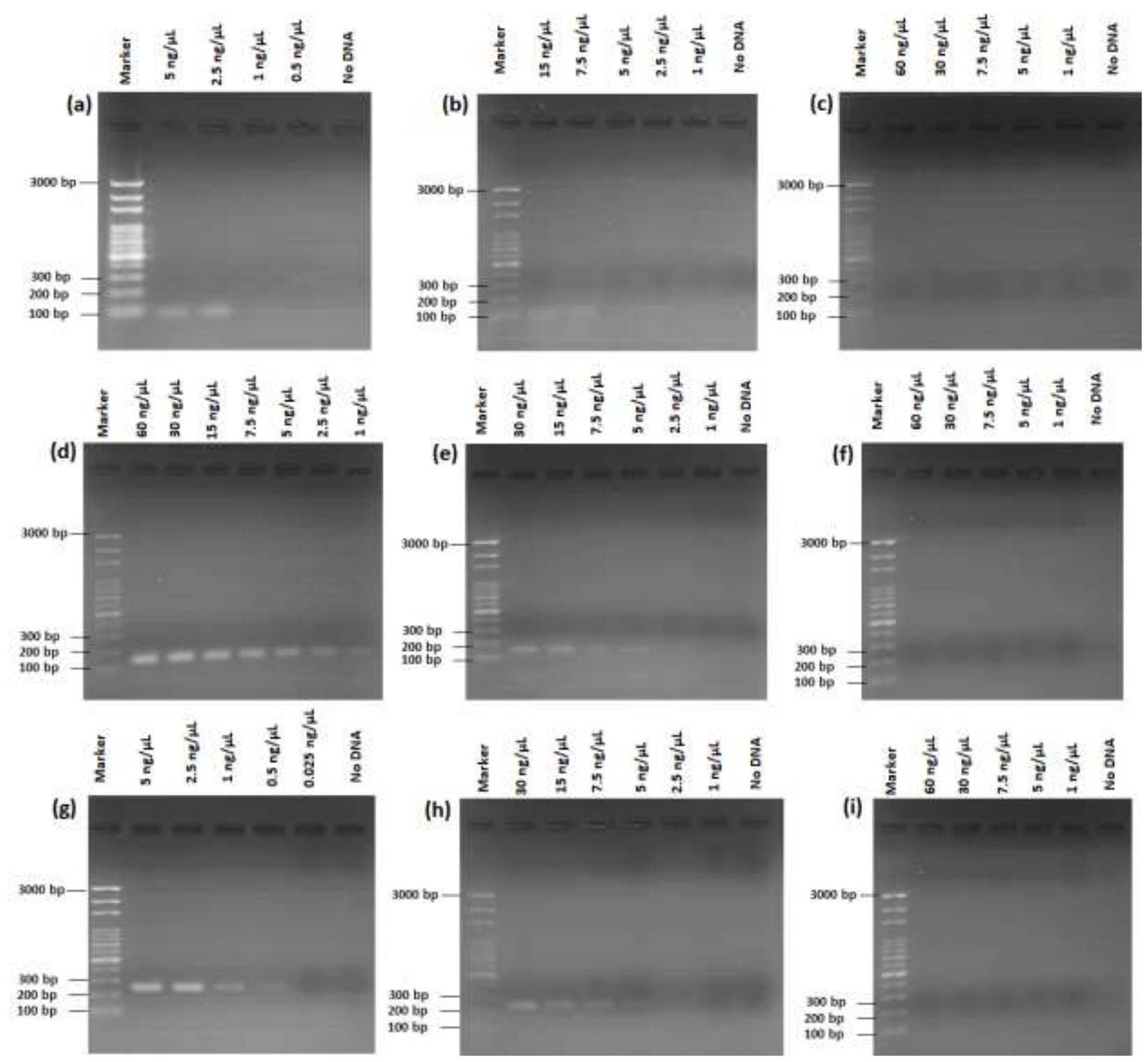

Figure S3. Gel electrophoresis image of $E$. coli DNA amplification of 84 bp (a-c), 147 bp (d-f) and 246 bp (g-i) amplicon for uidA gene (a) 30 PCR cycles. (b) 20 PCR cycles. (c) 10 PCR cycles. (d) 30 PCR cycles. (e) 20 PCR cycles. (f) 10 PCR cycles. (g) 30 PCR cycles. (h) 20 PCR cycles. (i) 10 PCR cycles. 
Table S1. Single PCR System

\begin{tabular}{|c|c|}
\hline Reagents & Concentration \\
\hline Sample & $1 \mu \mathrm{L}$ \\
\hline Forward $(10 \mu \mathrm{M})$ & $0.5 \mu \mathrm{L}$ \\
\hline Reverse $(10 \mu \mathrm{M})$ & $0.5 \mu \mathrm{L}$ \\
\hline PCR master mix & $12.5 \mu \mathrm{L}$ \\
\hline $\mathrm{ddH}_{2} \mathrm{O}$ added to reach & $25 \mu \mathrm{L}$ \\
\hline
\end{tabular}

\section{Milk sample analysis:}

Milk sample was purchased from the local market and used as test samples. Initially, centrifugation was done twice at $8000 \mathrm{rpm}$ to remove the fat other suspended particles in the milk. The sample was artificially inoculated with E. coli and cultured for several hours. $2 \mathrm{~mL}$ aliquot of the inoculated sample was centrifuged at $5000 \mathrm{rpm}$ for $3 \mathrm{~min}$ and the bacterial cells were extracted and re-dissolved in molecular biology grade water and used for PCR reaction. 5 $\mu \mathrm{L}$ samples were directly used for PCR reaction. Gel electrophoresis was used to confirm the DNA amplification (Figure S4a). Figure S4b shows a relative statistically significant difference $(\mathrm{p}<0.001, \mathrm{n}=3)$ in the correlation time between the artificially inoculated samples with $E$. coli cells in comparison with the control (without spiking E. coli cells), indicating the successful detection of E. coli for three different amplicons. This preliminary test confirms the potential of rotational diffusometry for future use of routine food microbial analysis. 

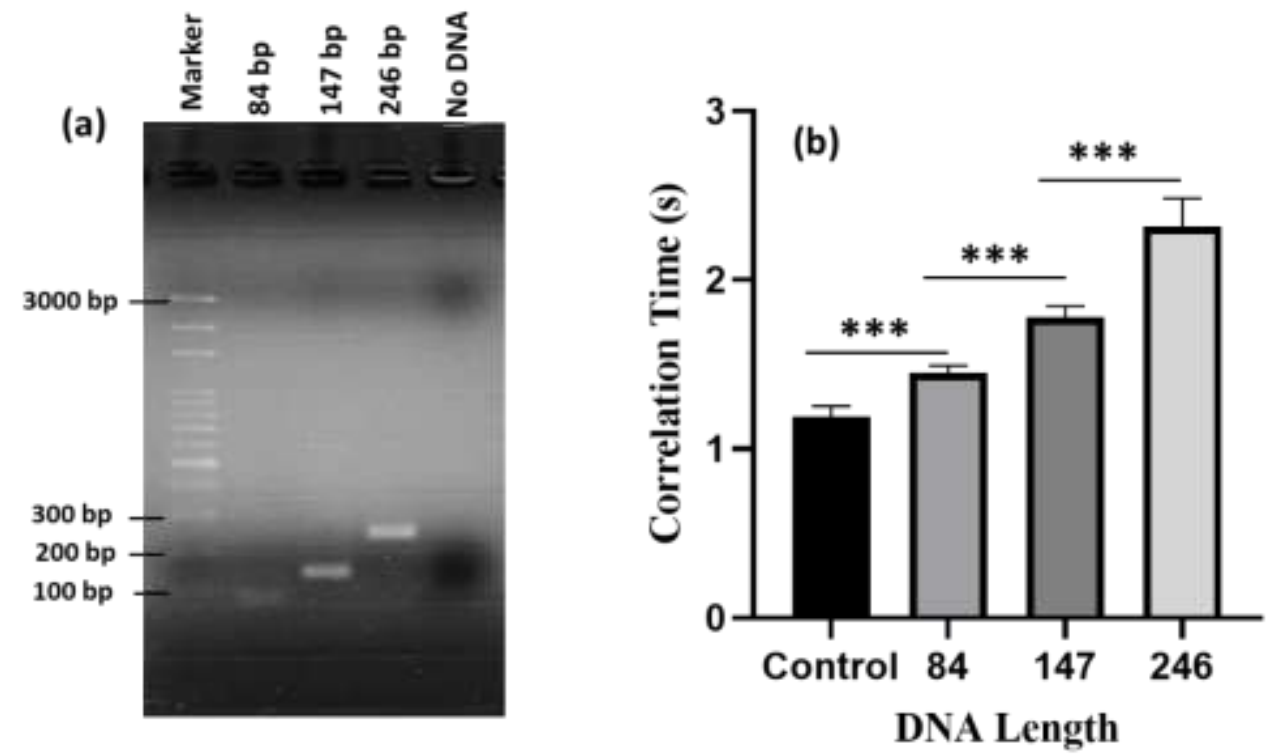

Figure S4: (a) Gel electrophoresis image of PCR amplification of E. coli whole cells using in artificially inoculated milk with three different amplicon - 84, 147 and 246 bp. (b) Rotational diffusometry measurements of correlation time change for PCR amplification of E. coli whole cells using in artificially inoculated milk with three different amplicon - 84, 147, and 246 bp. $(* * * \mathrm{p}<0.001, \mathrm{n}=3)$.

\section{Bacterial detection range in clinical samples:}

Urinary tract infections (UTI) are among the most common bacterial infections, majorly caused by E. coli, and the bacterial detection in clinical samples is normally reported in CFU (colony forming unit) per milliliter. Bacterial counts of $>10^{5} \mathrm{CFU} / \mathrm{mL}$, considered as positive for UTI ${ }^{1}$. In the present work, isolated bacterial DNA is used for all the experiments. Therefore, the comparison between DNA concentration and CFU count is very difficult. However, here we try to theoretically count the bacterial cells from the experimental DNA concentration. Since 1 bacterial cell contains $\sim 5$ fg of DNA and there is one copy of uidA gene per genome, therefore, 
the theoretical converted value of $50 \mathrm{pg} / \mu \mathrm{L}$ comes around $10^{7}$ cells $/ \mathrm{mL}$. However, the practical $\mathrm{CFU} / \mathrm{mL}$ value will largely vary from the theoretical value. Unfortunately, this calculated value is significantly higher than the clinically relevant value of bacterial counts for urinary tract infections. We look forward to working more in this and improve our methodology and perform a detailed study of bacterial detection in clinical samples as well."

\section{Supporting video:}

Video S1: Comparison of particle rotation in control and sample containing PCR amplified DNA under fluorescent microscope 40× objective lens.

\section{References:}

1. Wilson, M. L.; Gaido, L. Laboratory Diagnosis of Urinary Tract Infections in Adult Patients, Clin. Infect. Dis. 2004, 38 (8), 1150-1158 